\title{
miR-367 enhances the proliferation and invasion of cutaneous malignant melanoma by regulating phosphatase and tensin homolog expression
}

\author{
JIANWEN LONG ${ }^{1}$, JING LUO ${ }^{2}$ and XUWEN YIN ${ }^{2}$ \\ ${ }^{1}$ Department of Dermatology, The First Clinical School, Hubei University of Chinese Medicine; ${ }^{2}$ Department of \\ Dermatology, Hubei Provincial Hospital of Traditional Chinese Medicine, Wuhan, Hubei 430061, P.R. China
}

Received September 17, 2017; Accepted January 17, 2018

DOI: $10.3892 / \mathrm{mmr} .2018 .8663$

\begin{abstract}
Melanoma presents a serious threat to human health but the underlying mechanisms have not been fully identified. Increasing evidence indicates that microRNAs exert a significant influence on the tumorigenesis and metastasis of different types of cancer. The present study aimed to identify the role of microRNA (miR)-367 in the initiation and progression of melanoma and investigate its potential target. A total of 50 melanoma samples and 25 benign nevi tissues were used in the present study. Reverse transcription-quantitative polymerase chain reaction and western blot analysis were performed to determine the expression of mRNA and protein, respectively. Cell proliferation and invasion were assessed by $\mathrm{CCK}-8$, wound healing and Transwell assays. A proposed target mRNA of miR-367 was determined using a luciferase reporter assay and an in vivo xenograft model was used to evaluate the function of miR-367 in the progression of melanoma. It was revealed that miR-367 was overexpressed in melanoma tissues and cell lines. The miR-367 level in tumor tissues was positively correlated with tumor thickness, tumor stage, lymph node involvement, distant metastasis and the patient survival rate. A high miR-367 level was observed to enhance the growth, migration and invasion of melanoma cells. Conversely, low miR-367 levels suppressed the proliferation and invasion of melanoma cells. Furthermore, miR-367 was revealed to bind directly to phosphatase and tensin homolog (PTEN) mRNA and regulate the expression of the PTEN protein. miR-367 markedly increased the growth and invasion of melanoma cells, whereas the cotransfection of PTEN vectors limited the promoting function of miR-367 in the proliferation and invasion of A375 cells. The upregulation of miR-367 promoted tumor growth in vivo. In conclusion,
\end{abstract}

Correspondence to: Dr Jianwen Long, Department of Dermatology, The First Clinical School, Hubei University of Chinese Medicine, 4 Huayuanshan, Wuhan, Hubei 430061, P.R. China E-mail: longjianwen@hbhtcm.com

Key words: melanoma, microRNA, phosphatase and tensin homolog, proliferation, invasion the results revealed that miR-367 serves a positive role in the development of melanoma and may be an important target for the treatment of cutaneous melanoma.

\section{Introduction}

Cutaneous melanoma, developing in melanocytes that produce melanin, is a most pernicious tumors in skin malignancy and have a poor prognosis for survival (1). Emerging evidences indicate that melanoma gives rise to the majority of skin tumor-associated death (2-4), incontrollable growth and metastasis early are the main characteristics in the progression of cutaneous melanoma (5). The etiology of cutaneous melanoma is multifactorial and complicated including environmental and genetic factors $(6,7)$. Therefore, it is extremely crucial that further investigation of gene expression and regulation in the development of melanoma. miRNAs are small and noncoding RNA molecules which play key roles in post-transcriptional regulation of gene expression by binding to the 3'-UTR of targeted mRNA (8-10). Previous studies manifested microRNAs were involved in a range of biological activities including cell proliferation, transformation, and tumorigenesis (11). Emerging evidences identified that abnormal miRNAs expression was associated with the initiation and procession of tumors (12-14).

PTEN, known as phosphatase and tensin homolog deleted on chromosome 10 , is a phosphoinositide phosphatase that plays a tumor suppressor role in malignant melanoma (15). Increasing evidences revealed that microRNAs were involved in the tumorigenesis of tumors by regulating the expression of PTEN. For example, miR-500a promoted the invasion and migration of hepatocarcinoma via suppressing the expression of PTEN (16). miR-20b enhanced prostate cancer growth through targeting PTEN (17). So far, the regulatory relationship remains obscure and needs to be elucidated carefully between microRNAs and PTEN in malignant cutaneous melanoma.

miR-367 had been reported to be involved in the tumorigenesis and transformation of different cancers $(18,19)$, and it played tumor promotor or tumor suppressor roles in diverse tumors. For instance, overexpression of miR-367 was associated with a unfavorable prognosis and enhanced non-small cell lung cancer growth by binding to FBXW7 (20). In 
contrast, miR-367 was found to be related with TNM stage of patients and suppress gastric cancer development via targeting Rab23 (21). Up to date, the effect of miR-367 on cutaneous melanoma remains unclear. The present report aims to detect the expression of miR-367 in cutaneous melanoma tissues and cell lines, and investigate the regulatory mechanism of miR-367 on the development of cutaneous melanoma.

\section{Materials and methods}

Patients and tissues. Fifty melanoma tissues and 25 benign nevi tissues were collected from the Affiliated Hospital of Hubei University of Chinese Medicine and these samples were immediately frozen in liquid nitrogen until use. Written informed consents were obtained from all of recipients enrolled. These patients did not receive any radiation or chemotherapy before operation. All tissue sections were reviewed at least by two pathologists. The use of these specimens was approved by the Ethics Committee of the Affiliated Hospital of Hubei University of Chinese Medicine.

Cell culture. Human melanoma cell lines A375, WM35, SK-MEL-5 and SK-MEL-2 were incubated in Dulbecco's modified Eagle's medium supplemented with $10 \%$ fetal bovine serum (FBS; Gibco; Thermo Fisher Scientific, Inc., Waltham, MA, USA), $100 \mathrm{U} / \mathrm{ml}$ penicillin and $100 \mathrm{U} / \mathrm{ml}$ streptomycin at $37^{\circ} \mathrm{C}$ in $5 \% \mathrm{CO}_{2}$ atmosphere. Human epidermal melanocytes (HEMa-LP) were obtained from Cascade Biologics, Inc. (Mansfield, UK) and were cultured in medium with HMGS-2 in a humidified atmosphere of $5 \% \mathrm{CO}_{2}$ at $37^{\circ} \mathrm{C}$.

Cell transfection. A375 cells were transiently transfected with Lipofectamine 2000 according to the manufacturer's instructions (Invitrogen; Thermo Fisher Scientific, Inc.). Cells were transfected with miR-367 mimics, miR-367 inhibitor, control vectors or pcDNA3.1 vector encoding PTEN (GeneCopoeia, Inc., Rockville, MY, USA). The transfected cells were incubated at $37^{\circ} \mathrm{C}$ and $5 \% \mathrm{CO}_{2}$. After 4 to $6 \mathrm{~h}$, the transfection solution was replaced with fresh culture medium for subsequent experiments.

RNA extraction and reverse transcription-quantitative polymerease chain reaction. The total RNA of the tissue samples and cells was extracted using the TRIzol reagent (Invitrogen; Thermo Fisher Scientific, Inc.) according to the manufacturer's instructions. cDNA was converted and amplified using the TaqMan miRNA reverse transcription kit according to the manufacturer's assay procedure. Quantitative real-time PCR was performed with TaqMan human MiRNA assay kit. miR-367 expression was calculated relative to the expression of U6. The expression changes were determined using $2^{-\Delta \Delta C t}$ method. miR-367 forward, 5'-TTCTCCGAACTTGTCACG TTT-3' and reverse, 5'-ACGTGACACGTTCGGAGAATT-3'; U6 forward, 5'-CTCGCTTCGGCAGCACA-3' and reverse, 5'-AACGCTTCACGAATTTGCGT-3'.

Cell proliferation assay. Melanoma cells proliferation was measured by using a cell proliferation kit, Cell Counting Kit-8, according to the manufacturer's instructions. Cells after transfection were seeded into 96-well culture plates and incubated at $37^{\circ} \mathrm{C}$. Fresh culture media $(90 \mu \mathrm{l})$ and $10 \mu \mathrm{l} \mathrm{CCK}-8$
Table I. Association between clinical findings and the miR-367 level in individuals with cutaneous melanoma.

\begin{tabular}{|c|c|c|c|c|}
\hline \multirow{2}{*}{$\begin{array}{l}\text { Clinicopathological } \\
\text { findings }\end{array}$} & \multirow{2}{*}{$\begin{array}{c}\text { Patients } \\
(n=50)\end{array}$} & \multicolumn{2}{|c|}{$\operatorname{miR}-367$} & \multirow[b]{2}{*}{ P-value } \\
\hline & & High & Low & \\
\hline \multicolumn{5}{|l|}{ Age (years) } \\
\hline$\leq 60$ & 30 & 17 & 13 & \\
\hline$>60$ & 20 & 8 & 12 & 0.387 \\
\hline \multicolumn{5}{|l|}{ Sex } \\
\hline Male & 28 & 15 & 13 & \\
\hline Female & 22 & 10 & 12 & 0.776 \\
\hline \multicolumn{5}{|c|}{ Tumor thickness (mm) } \\
\hline$\leq 1$ & 11 & 2 & 9 & \\
\hline$>1$ & 39 & 23 & 16 & 0.037 \\
\hline \multicolumn{5}{|l|}{ TNM classification } \\
\hline $\mathrm{I}+\mathrm{II}$ & 13 & 2 & 11 & \\
\hline III+IV & 37 & 23 & 14 & 0.008 \\
\hline \multicolumn{5}{|c|}{ Lyph node involvement } \\
\hline No & 17 & 4 & 13 & \\
\hline Yes & 33 & 21 & 12 & 0.016 \\
\hline \multicolumn{5}{|l|}{ Distant metastasis } \\
\hline No & 39 & 16 & 23 & \\
\hline Yes & 11 & 9 & 2 & 0.037 \\
\hline
\end{tabular}

miR, microRNA.

solutions were added to each sample at 12, 24, 48 and $72 \mathrm{~h}$. Subsequently, the transfected cells were cultured at $37^{\circ} \mathrm{C}$ for $2 \mathrm{~h}$. Cell growth was calculated by a microplate reader at a wave length of 450 nanometer.

Cell migration assay. Wound healing assay was used to study cell migration. Melanoma cells were seeded and incubated in 6-well plates until monolayers were developed. A plastic scriber was used to make an artificial wound, and the image of the wound was served as baseline. Cells were then washed twice with PBS and incubated in DMEM with $10 \%$ fetal bovine serum at $37^{\circ} \mathrm{C}$ for $48 \mathrm{~h}$. Cell migration capacity was assessed by subtracting the final wound width from the initial wound width.

Transwell invasion assay. Transwell invasion assay was performed to evaluate cell invasion ability. A Transwell chamber was precoated with Matrigel (BD Biosciences, Franklin Lakes, NJ, USA). Cells $\left(2 \times 10^{5}\right)$ were seeded into the upper chamber and incubated in serum free medium. The incubating media with $20 \%$ fetal bovine serum were added into the lower chamber to attract cells. After being cultured for $48 \mathrm{~h}$, cells on the upper chamber were cleared with a swab. Cells after migration were fixed and stained with $0.5 \%$ crystal violet, photographed and counted under a microscope.

Luciferase reporter analysis. The 3'-UTR region of PTEN or the mutated sequence was cloned into pMIR-REPORT vectors. miR-367 mimics, or corresponding control was transfected 
A

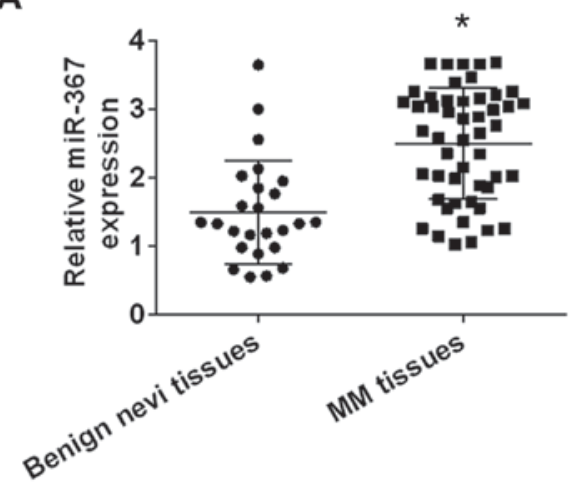

C

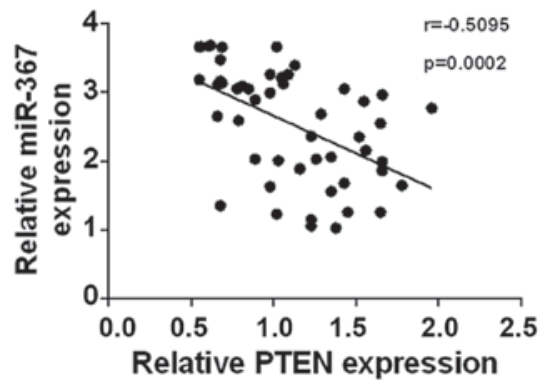

B

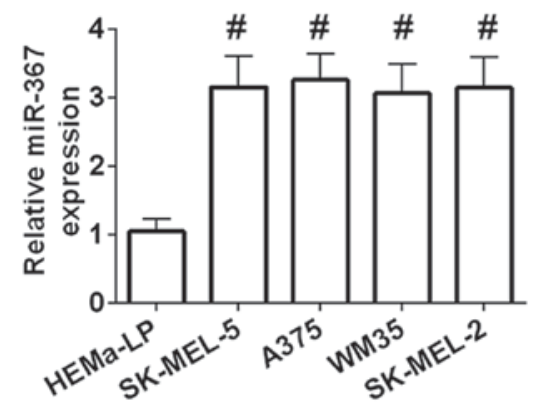

D

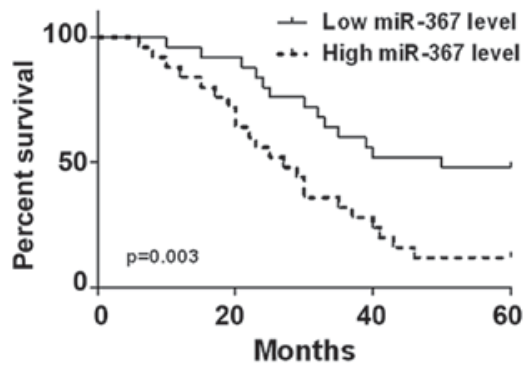

Figure 1. Overexpression of miR-367 in cutaneous melanoma tissues and cell lines. (A) miR-367 was overexpressed in cutaneous melanoma tissues. (B) The levels of miR-367 were significantly increased in SK-MEL-5 cells, A375 cells, WM35 cells, and SK-MEL-2 cells compared with HEMa-LP cells. (C) The miR-367 level was negatively correlated with the PTEN level in melanoma tissues. (D) High expression of miR-367 was positively correlated with decreased overall survival. ${ }^{*} \mathrm{P}<0.01$ compared with benign nevi tissues; ${ }^{\#} \mathrm{P}<0.01$ compared with HEMa-LP cells. miR, microRNA.

together with reporter plasmid and pRL-SV40 (Promega, Madison, WI, USA) into A375 cells. The active luciferase level was assessed $48 \mathrm{~h}$ after transfection by a Luciferase Reporter Assay (Promega).

Western blot analysis. The concentration of protein was determined by the BCA kit (Pierce, Rockford, IL USA). SDS-PAGE assay was performed to separate the extracted protein mixtures. The proteins were transferred to a PVDF membrane and incubated with the corresponding antibodies. Antibodies listed below were used to determine the protein expression: PTEN (1:500; Santa Cruz Biotechnology, Inc., Dallas, TX, USA), AKT (1:500), and p-AKT (1:500) (both from Abcam, Cambridge, MA, USA). Anti-GAPDH (1:2,000; Sigma-Aldrich; Merck KGaA, Darmstadt, Germany). Horseradish peroxidase-conjugated secondary antibody $(1: 2,000$; Abcam). Band intensity was evaluated using an ECL chemiluminescent kit (Millipore, Billerica, MA, USA).

Tumor growth in vivo. Nude mice were prepared to determine the melanoma growth (25-30 g, 6-week-old), A375 cells after transfection were harvested by centrifugation, washed with PBS and re-suspended, A375 cells were subcutaneously injected into nude mice $(n=6)$, mice were sacrificed on 5, 10, 15, 20 and 25 days, and tumor tissues were excised for further study. The weight and volume of the tumor mass were measured and recorded.

Statistical analysis. SPSS 16.0 (SPSS, Inc., Chicago, IL, USA) was performed for statistical analyses, including t-test, one-way analysis of variance with post hoc contrasts by
Student-Newman-Keuls test, Fisher exact probability test, Pearson correlation analysis and Kaplan-Meier plot. All data were represented as mean \pm standard deviation. $\mathrm{P}<0.05$ was considered to indicate a statistically significant difference.

\section{Results}

Overexpression of miR-367 was confirmed in cutaneous melanoma and was correlated with poor prognosis in patients with cutaneous melanoma. qRT-PCR was used to determine the expression of miR-367 in 50 of cutaneous melanoma tissues and 25 of benign nevi tissue. Our results identified that the miR-367 level was significantly elevated in melanoma tissues in comparison with benign nevi tissues (Fig. 1A). High expression of miR-367 was confirmed in A375 cells, WM35 cells, SK-MEL-2 cells and SK-MEL-5 cells compared with HEMa-LP cells, and the highest level of miR-367 was found in A375 cells line (Fig. 1B). The miR-367 level was negatively correlated with the PTEN level in melanoma tissues (Fig. 1C). Patients with melanoma were separated into high miR-367 expression group $(n=25)$ and low miR-367 expression $(n=25)$ according to the relative median value of miR-367 (median, 2.665; Min-Max, 1.030-3.680). The correlation was analyzed between the miR-367 expression and clinical characteristics of patients with melanoma. The correlation was not statistically significant between the miR-367 level and age, sex of patients with melanoma, but high expression of miR-367 was significantly correlated with tumor thickness, TNM stage, lymph node involvement and distant metastasis of malignant melanoma as shown in Table I. Besides, high miR-367 level was positively correlated with decreased overall survival (Fig. 1D). 
A

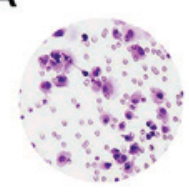

miR-control

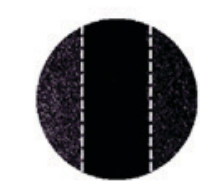

$\mathbf{O h}$

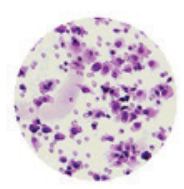

miR-367 mimics

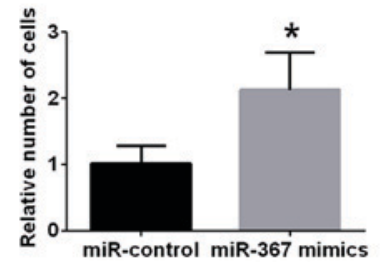

miR-control miR-367 mimics

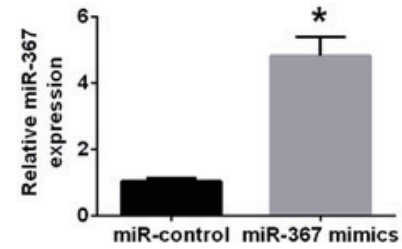

miR-control miR-367 mimics
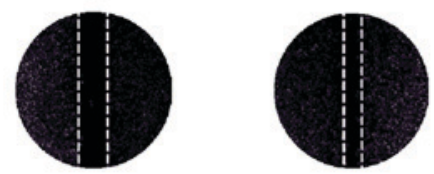

miR-control

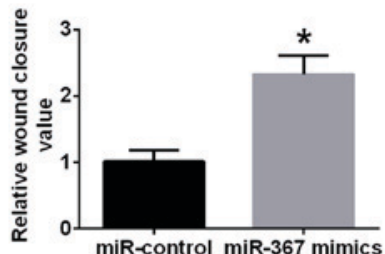

miR-control miR-367 mimics

B

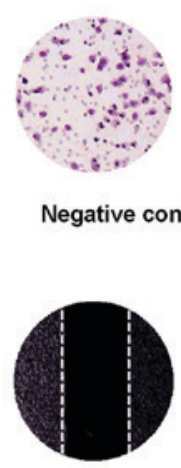

$\mathrm{Oh}$
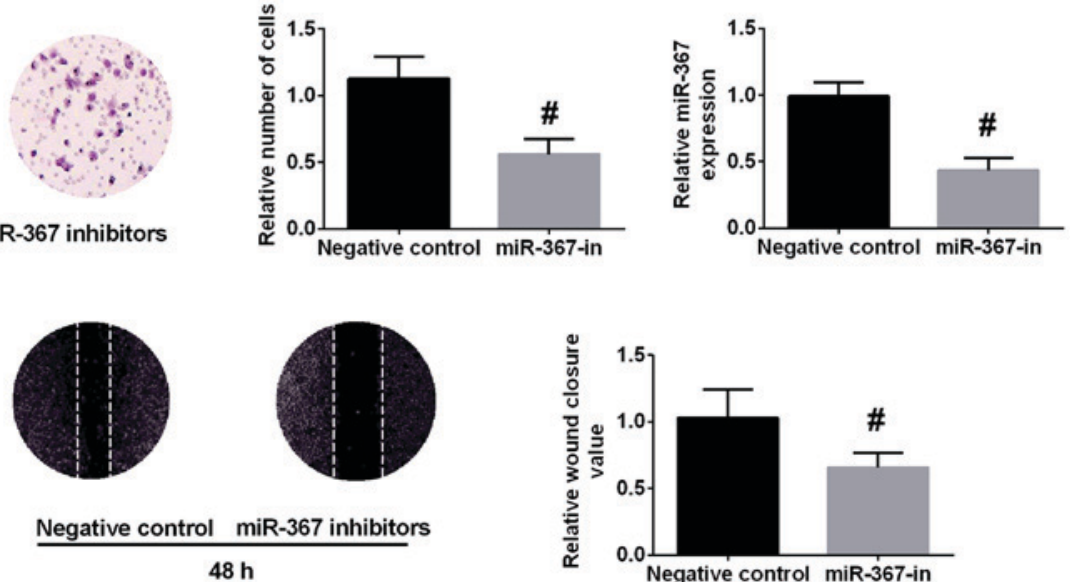

Figure 2. Upregulation of miR-367 promotes A375 cell invasion and migration. (A) The miR-367 level in A375 cells was overexpressed in miR-367 mimics group. High level of miR-367 markedly enhanced A375 cells invasion and migration. (B) miR-367 inhibitors significantly decreased the level of miR-367 in A375 cells. Downregulation of miR-367 distinctly attenuated A375 cells invasion and migration. ${ }^{*} \mathrm{P}<0.05$, compared with miR-control group; ${ }^{\#} \mathrm{P}<0.05$, compared with negative control group. miR, microRNA.

The results manifested that miR-367 played a promoting role in the development of melanoma.

High level of miR-367 enhanced the growth and invasion of A375 cells. A375 cells were selected for further experiments due to the highest level of miR-367 in A375 cells. The miR-367 level was significantly upregulated in miR-367 mimics group in comparison with miR-control group (Fig. 2A). CCK-8 assay, Transwell assays and wound healing assay confirmed that upregulation of miR-367 distinctly promoted the proliferation, invasion and migration of A375 cells (Fig. 2A). Conversely, the miR-367 level was decreased in A375 cells after transfection with miR-367 inhibitor (Fig. 2B). The transfection of miR-367 inhibitor attenuated the proliferation, invasion and migration of A375 cells (Fig. 2B). These data identified that miR-367 play a critical role in the growth, invasion and migration of A375 cells.

PTEN mRNA was identified as the functional downstream target of miR-367. Bioinformatics analysis (TargetScan 7.0 and miRanda) was performed to investigate the downstream targets of miR-367 in melanoma cells. Retrieval results manifested that 3'-UTR of PTEN contained a direct binding sequence of miR-367 (Fig. 3), miR-367 interaction with binding sequence of 3'-UTR-PTEN was confirmed by luciferase reporter assay. The results indicated that miR-367 distinctly inhibited luciferase activity of PTEN in wild-type, however, had no effect on that of PTEN in mutated type (Fig. 3B). Western blot analysis was implemented to identify whether miR-367 could regulate PTEN expression in A375 cells or not. The results further identified that high level of miR-367 remarkably suppressed PTEN expression in A375 cells. Furthermore, the phosphorylation of AKT was increased by the transfection of miR-367 mimics into A375 cells (Fig. 4A). On the other hand, the PTEN expression was significantly elevated and the phosphorylation of AKT was downregulated in A375 cells after transfection with miR-367 inhibitors (Fig. 4B). Collectively, the results identified that PTEN was an important downstream targeting mRNA of miR-367 in A375 cells.

Upregulation of PTEN attenuated the effect of miR-367 on the proliferation and invasion of A375 cells. The transfection of PTEN vectors significantly increased the expression of PTEN in PTEN vectors group compared with empty vectors 
A

Hsa-miR-367 3'-AgugguaAcGauUucacguUaA-5'

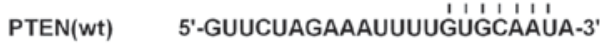

PTEN(mut) 5'-GUUCUAGaAaUUUUCACGUUAA-3'

B

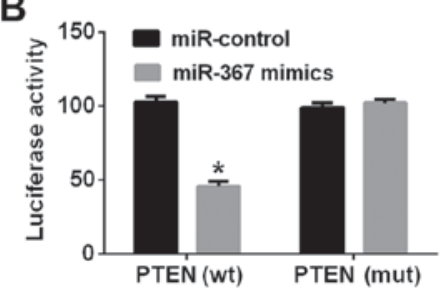

E

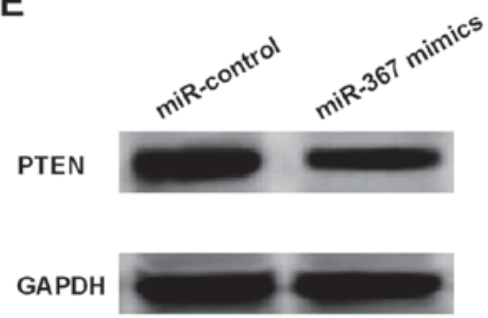

C

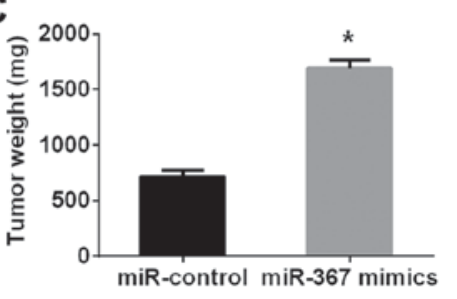

$\mathbf{F}$

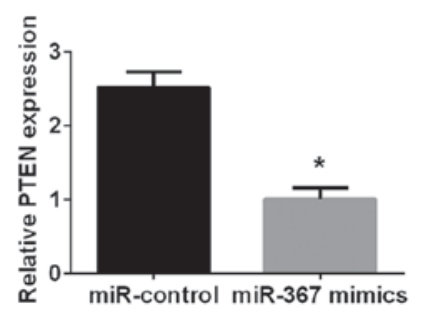

D

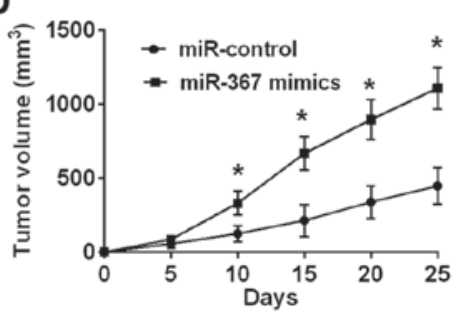

G

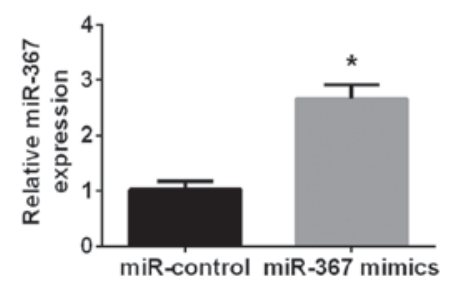

Figure 3. PTEN mRNA is a specific binding molecule of miR-367 in A375 cells. (A) miR-367 and its predicted targeting region for wild PTEN. (B) miR-367 suppressed luciferase reporter activities of PTEN in wild-type, but had no significant effect on that of PTEN in mutated type. (C) Tumor weight was markedly raised in miR-367 group compared with miR-control group. (D) Tumor volume was significantly increased in miR-367 group compared with miR-control group. (E) PTEN expression was shown in xenograft tumors. (F) PTEN expression in miR-367 mimics group was distinctly lower than that in miR-control group. (G) The level of miR-367 was elevated in miR-367 mimics group compared with miR-control group. "P<0.05, compared with miR-control group. miR, microRNA; PTEN, phosphatase and tensin homolog.

A

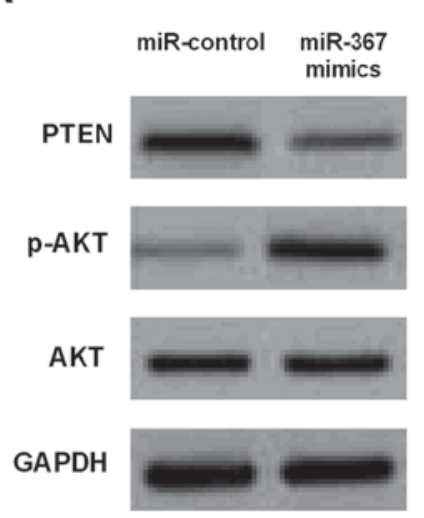

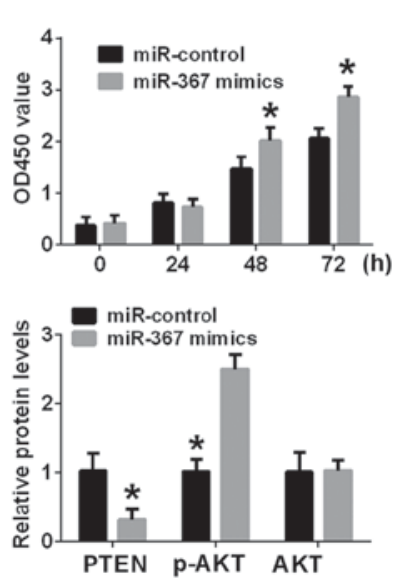

C

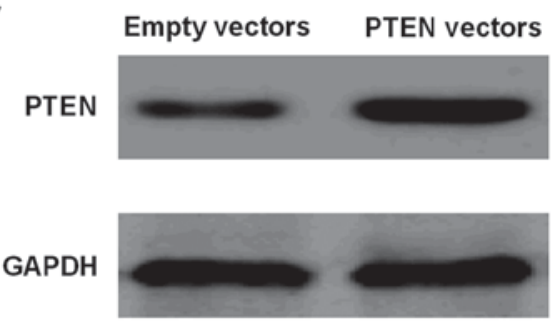

B

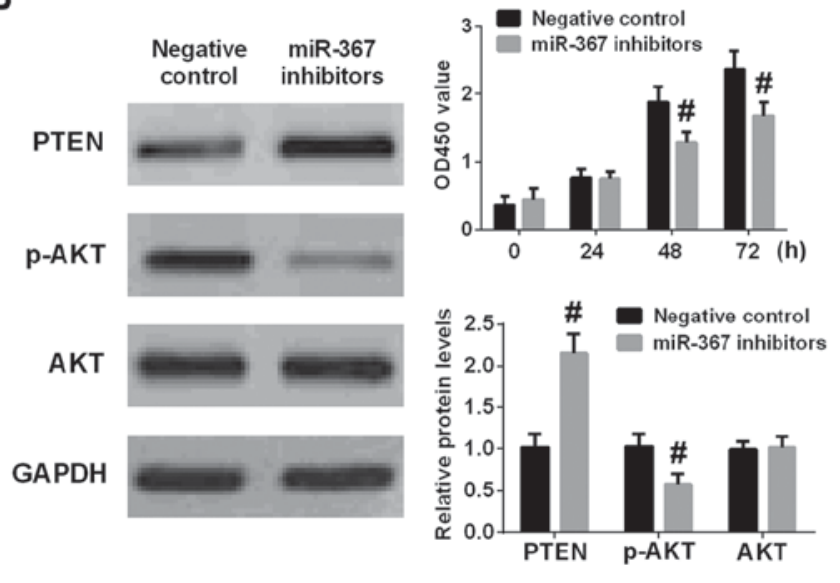

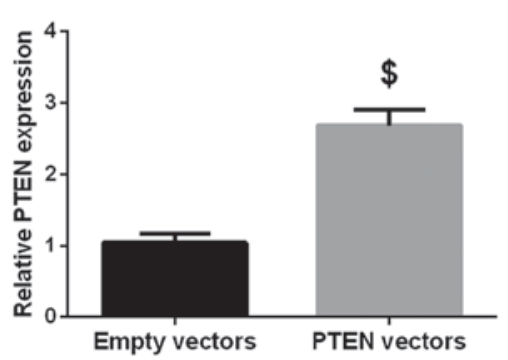

Figure 4. miR-367 promotes PTEN expression and inhibits the phosphorylation of AKT in A375 cells, and enhanced cell proliferation. (A) Upregulation of miR-367 distinctly reduced PTEN level, promoted the phosphorylation of AKT, and increased cell proliferation. (B) Downregulation of miR-367 markedly raised the PTEN level, attenuated the phosphorylation of AKT and inhibited cell proliferation. (C) The PTEN level was significantly raised in A375 cells transfected with PTEN vectors in comparison with empty vectors group. ${ }^{*} \mathrm{P}<0.01$, compared with miR-control group; $\mathrm{P}<0.01$, compared with negative control group; ${ }^{\$} \mathrm{P}<0.01$, compared with empty vectors group. miR, microRNA; PTEN, phosphatase and tensin homolog; AKT, protein kinase B. 

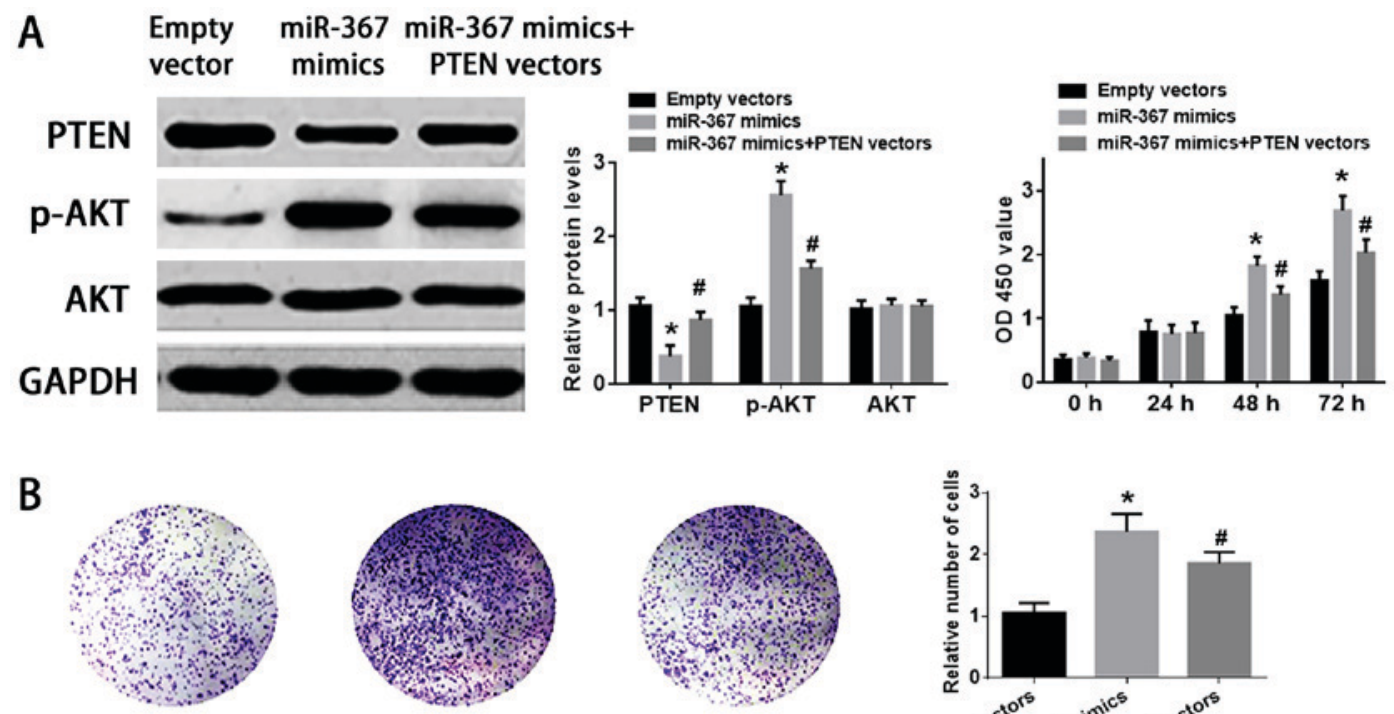

Empty vectors
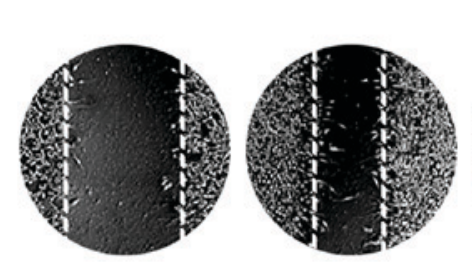

Empty vectors
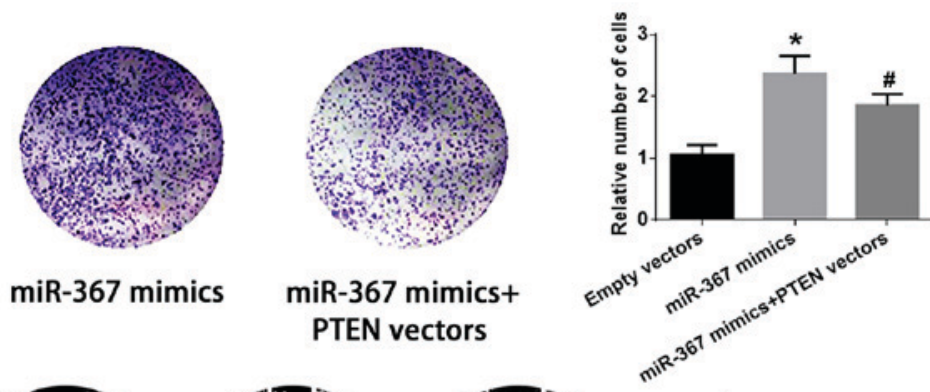

$\mathbf{O h}$

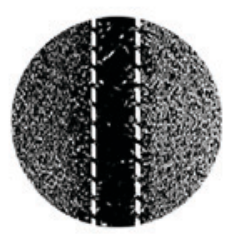

miR-367 mimics

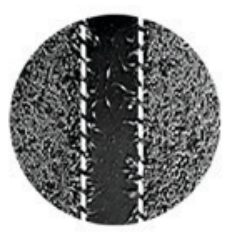

miR-367 mimics+ PTEN vectors

$48 \mathrm{~h}$

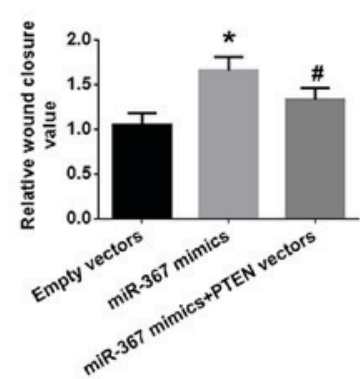

Figure 5. Forced expression of PTEN attenuated the enhancing effect of miR-367 on the proliferation and migration of A375 cells. (A) Transfection of miR-367 inhibited PTEN expression, increased the phosphorylation of AKT and increased cell growth in A375 cells, but the cotransfection of PTEN vectors attenuated these functions of miR-367 mimics in A375 cells. (B) Forced expression of miR-367 mimics enhanced the invasion and migration of A375 cells, but upregulation of PTEN limited partially the ability of invasion and migration of A 375 cells transfected with miR-367 mimics. "P $<0.01$ compared with empty vectors group; " $\mathrm{P}<0.05$ compared with miR-367 mimics group. miR, microRNA; PTEN, phosphatase and tensin homolog; AKT, protein kinase B.

group (Fig. 4C). The transfection of miR-367 mimics remarkably suppressed the expression of PTEN and enhanced the phosphorylation of AKT in A375 cells, but the cotransfection of miR-367 mimics with PTEN vectors attenuated the effect of miR-367 mimics on the expression of PTEN and the phosphorylation of AKT in A375 cells. In addition, upregulation of miR-367 enhanced the growth, invasion and migration of A375 cells, however, PTEN vectors ameliorated the ability of growth, invasion, and migration of A375 cells transfected with miR-367 mimics. Finally, PTEN vectors and miR-367 mimics had no significant effect on AKT level in A375 cells (Fig. 5). Briefly, miR-367 was able to promote the proliferation, invasion and migration of A375 cells via targeting PTEN.

The effect of miR-367 on melanoma growth in a xenograft tumor model. A xenograft tumor model was applied to confirm the promoting function of miR-367 in the progression of melanoma. The xenograft tumors were collected for determination of weight and volume. The result showed that the volume of tumor in miR-367 mimics group was significantly increased in comparison with miR-control group at 10, 15, 20 and 25 days after A375 cells injection (Fig. 3D), and the weight of tumor in miR-367 mimics group was markedly larger than that in miR-control group at 25 days after A375 cells injection
(Fig. 3C). Therefore, it was identified strongly that forced expression of miR-367 markedly augmented the growth of xenograft tumors. In addition, the PTEN expression in tissues was significantly decreased, but the miR-367 level in tissues was distinctly increased in miR-367 mimics group compared with miR-control group (Fig. 3E-G). Accordingly, enhancing function of miR-367 in the malignant melanoma growth was confirmed in vivo.

\section{Discussion}

miRNA has been identified as a critical transcriptional mediator in many biological activities including the development of tumorgenesis by binding to 3'-UTR of its targeting mRNA. The aberrant expression of miR-367 was found definitely in many types of tumors $(22,23)$. The association between miR-367 and cutaneous malignant melanoma has not been clearly elucidated up to date. Accordingly, we investigated the expression and underlying regulatory function of miR-367 in malignant melanoma. As a result, we found that miR-367 was distinctly overexpressed in cutaneous melanoma tissues and cell lines, and negatively correlated with the PTEN level in cutaneous melanoma tissues. High expression of miR-367 was markedly related with the malignant clinical characteristics of 
individuals with melanoma. Next, overexpression of miR-367 enhanced A375 cells proliferation and Transwell migration, and inhibited PTEN expression, upregulation of PTEN alleviated partially the enhancing effect of miR-367 on the growth, invasion and migration of A375 cells. Finally, nude mice models study identified in vivo that overexpression of miR-367 promoted xenograft tumor development. All of these results combined manifested that miR-367 promoted melanoma progression by, at least in part, regulating the PTEN expression.

PTEN is a crucial tumor inhibitor gene that is involved in the occurence and progression of many types of malignant tumor (24) including malignant melanoma (25). The protein derived from the gene can reduce phosphatidylinositol-3,4,5-trisphosphate level and exert a tumor inhibitor role through PTEN/AKT pathway (26). In this study, PTEN was identified to be an important functional target of miR-367 in melanoma cells. Firstly, the binding target of miR-367 was discovered in 3'UTR-PTEN mRNA. Secondly, miR-367 decreased distinctly the luciferase activity in the wild PTEN, however, did not affect luciferase activity in the mutated PTEN. Thirdly, forced expression of miR-367 inhibited expression of PTEN and promoted the phosphorylation of AKT, whereas downregulation of miR-367 distinctly promoted the expression of PTEN and alleviated the phosphorylation of AKT. Upregulation of PTEN suppressed the pro-tumor function of miR-367 in A375 cells. Finally, The PTEN expression was distinctly reduced but the miR-367 level was markedly raised in xenograft tumors in miR-367 mimics group in comparison with miR-control group. All results combined identified that miR-367 played a pro-tumor role in A375 cells via regulating PTEN expression. PTEN was a potential mediator of the PTEN/PI3K/AKT pathway (27). These results further confirmed miR-367 could regulate the phosphorylation of AKT level by targeting PTEN mRNA, and be involved in malignant behaviors of A375 cells via regulating PTEN/AKT pathway.

Together, this study confirms the miR-367 level is distinctly upregulated in cutaneous melanoma. Overexpression of miR-367 is correlated with malignant clinical characteristics of individuals with cutaneous melanoma. miR-367 augments the proliferation and invasion of melanoma. Furthermore, miR-367 plays promoting roles on the development of melanoma via modulating PTEN expression. Regulation of miR-367 expression may be a novel and crucial strategy for cutaneous melanoma treatment.

\section{References}

1. Isola AL, Eddy K and Chen S: Biology, therapy and implications of tumor exosomes in the progression of melanoma. Cancers 8: E110, 2016.

2. Giblin AV and Thomas JM: Incidence, mortality and survival in cutaneous melanoma. J Plast Reconstr Aesthet Surg 60: 32-40, 2007.

3. Peterson M, Albertini MR and Remington P: Incidence, survival and mortality of malignant Cutaneous melanoma in Wisconsin, 1995-2011. WMJ 114: 196-201, 2015.

4. Downing A, Newton-Bishop JA and Forman D: Recent trends in cutaneous malignant melanoma in the Yorkshire region of England; incidence, mortality and survival in relation to stage of disease, 1993-2003. Br J Cancer 95: 91-95, 2006.

5. Leiter U, Meier F, Schittek B and Garbe C: The natural course of cutaneous melanoma. J Surg Oncol 86: 172-178, 2004.
6. Luo C, Weber CE, Osen W, Bosserhoff AK and Eichmuller SB: The role of microRNAs in melanoma. Eur J Cell Biol 93: 11-22, 2014.

7. Kashani-Sabet M: Molecular markers in melanoma. Br J Dermatol 170: 31-35, 2014.

8. Mannucci C, Casciaro M, Minciullo PL, Calapai G, Navarra M and Gangemi S: Involvement of microRNAs in skin disorders: A literature review. Allergy Asthma Proc 38: 9-15, 2017.

9. Greenberg E, Nemlich Y and Markel G: MicroRNAs in cancer: Lessons from melanoma. Curr Pharm Des 20: 5246-5259, 2014.

10. Carthew RW: Gene regulation by microRNAs. Curr Opin Genet Dev 16: 203-208, 2006.

11. Nakahara K and Carthew RW: Expanding roles for miRNAs and siRNAs in cell regulation. Curr Opin Cell Biol 16: 127-133, 2004.

12. Long J, Menggen Q, Wuren Q, Shi Q and Pi X: miR-219-5p inhibits the growth and metastasis of malignant melanoma by targeting BCL-2. Biomed Res Int 2017: 9032502, 2017.

13. He J, Zhao J, Zhu W, Qi D, Wang L, Sun J, Wang B, Ma X, Dai Q and $\mathrm{Yu} \mathrm{X}$ : MicroRNA biogenesis pathway genes polymorphisms and cancer risk: A systematic review and meta-analysis. Peer J 4: e2706, 2016.

14. Fattore L, Costantini S, Malpicci D, Ruggiero CF, Ascierto PA, Croce CM, Mancini R and Ciliberto G: MicroRNAs in melanoma development and resistance to target therapy. Oncotarget 8 : 22262-22278, 2017.

15. Stahl JM, Cheung M, Sharma A, Trivedi NR, Shanmugam S and Robertson GP: Loss of PTEN promotes tumor development in malignant melanoma. Cancer Res 63: 2881-2890, 2003.

16. Zhao Y, Wang Y and Wang Y: Up-regulated microRNA-500a enhances hepatocarcinoma metastasis by repressing PTEN expression. Biosci Rep 37: BSR20170837, 2017.

17. Guo J, Xiao Z, Yu X and Cao R: miR-20b promotes cellular proliferation and migration by directly regulating phosphatase and tensin homolog in prostate cancer. Oncol Lett 14: 6895-6900, 2017.

18. Guan Y, Chen L, Bao Y, Qiu B, Pang C, Cui R and Wang Y: High miR-196a and low miR-367 cooperatively correlate with unfavorable prognosis of high-grade glioma. Int J Clin Exp Pathol 8: 6576-6588, 2015

19. Sun J, Song K, Feng X and Gao S: MicroRNA-367 is a potential diagnostic biomarker for patients with esophageal squamous cell carcinoma. Biochem Biophys Res Commun 473: 363-369, 2016.

20. Xu J, Wu W, Wang J, Huang C, Wen W, Zhao F, Xu X, Pan X, Wang W, Zhu Q and Chen L: miR-367 promotes the proliferation and invasion of non-small cell lung cancer via targeting FBXW7. Oncol Rep 37: 1052-1058, 2017.

21. Bin Z, Dedong H, Xiangjie F, Hongwei X and Qinghui Y: The microRNA-367 inhibits the invasion and metastasis of gastric cancer by directly repressing Rab23. Genet Test Mol Biomarkers 19: 69-74, 2015.

22. Campayo M, Navarro A, Vinolas N, Diaz T, Tejero R, Gimferrer JM, Molins L and Cabanas ML: Low miR-145 and high miR-367 are associated with unfavourable prognosis in resected nonsmall cell lung cancer. Eur Respir J 41: 1172-1178, 2013.

23. Kaid C, Silva PB, Cortez BA, Rodini CO, Semedo-Kuriki P and Okamoto OK: miR-367 promotes proliferation and stem-like traits in medulloblastoma cells. Cancer Sci 106: 1188-1195, 2015.

24. Milella M,Falcone I, Conciatori F, Cesta Incani U, Del Curatolo A, Inzerilli N, Nuzzo CM, Vaccaro V, Vari S, Cognetti F and Ciuffreda L: PTEN: Multiple functions in human malignant tumors. Front Oncol 5: 24, 2015.

25. Yang H, Kircher DA, Kim KH, Grossmann AH, VanBrocklin MW, Holmen SL and Robinson JP: Activated MEK cooperates with Cdkn2a and Pten loss to promote the development and maintenance of melanoma. Oncogene 36: 3842-3851, 2017.

26. Song MS, Salmena L and Pandolfi PP: The functions and regulation of the PTEN tumour suppressor. Nat Rev Mol Cell Biol 13: 283-296, 2012.

27. Carnero A, Blanco-Aparicio C, Renner O, Link W and Leal JF: The PTEN/PI3K/AKT signalling pathway in cancer, therapeutic implications. Curr Cancer Drug Targets 8: 187-198, 2008.

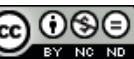

This work is licensed under a Creative Commons Attribution-NonCommercial-NoDerivatives 4.0 International (CC BY-NC-ND 4.0) License. 